iSCAT-Mikroskopie

\title{
Interferenz von Licht macht einzelne unmarkierte Proteine sichtbar
}

\author{
KATHARINA KÖNIG ${ }^{1}$, ANDRÉ GEMEINHARDT ${ }^{1}$, VAHID SANDOGHDAR ${ }^{1,2}$ \\ ${ }^{1}$ MAX-PLANCK-INSTITUT FÜR DIE PHYSIK DES LICHTS, ERLANGEN \\ 2 DEPARTMENT PHYSIK, FRIEDRICH-ALEXANDER-UNIVERSITÄT ERLANGEN- \\ NÜRNBERG, ERLANGEN
}

iSCAT microscopy is a powerful tool for the optical detection and visualization of small nanoparticles, down to single unlabeled proteins. In this overview, we give an introduction to the method's principles and benefits and show how it can be applied to monitor protein secretion of single living cells with single-protein sensitivity at millisecond temporal resolution.

DOI: $10.1007 / \mathrm{s} 12268-019-0225-9$

(C) Die Autoren 2019

Der menschliche Körper ist ein komplexer Organismus, dessen Funktionsweise auf einer äußerst vielschichtigen und detaillierten Kommunikation verschiedenster Zelltypen über eine Vielfalt an Botenstoffen beruht. Um diese Prozesse $\mathrm{zu}$ verstehen und möglichen Fehlfunktionen vorzubeugen oder diese zu behandeln, ist es notwendig, die zellulären
Abläufe im Detail zu untersuchen. Eine kritische Komponente stellt dabei die Sekretion von Proteinen durch (stimulierte) Zellen dar, die beispielsweise innerhalb des Immunsystems eine wichtige Rolle spielt. Hier können diese Proteine von anderen Zellen wahrgenommen werden und verschiedene Reaktionen und Prozesse in der Immunabwehr auslösen.

Die Untersuchung solcher Sekretionsprozesse birgt einige Herausforderungen. Traditionelle Methoden, wie Massenspektrometrie, antikörperbasierte Nachweisverfahren (z. B. ELISA) oder Durchflusscytometrie (z. B. FACS), sind sehr aufwendig, langwierig und benötigen das Markieren der Probe mittels Isotopen oder fluoreszenten Molekülen. Das Markieren von Proteinen ist weitverbreitet und für einen Großteil der biologisch moti-
A

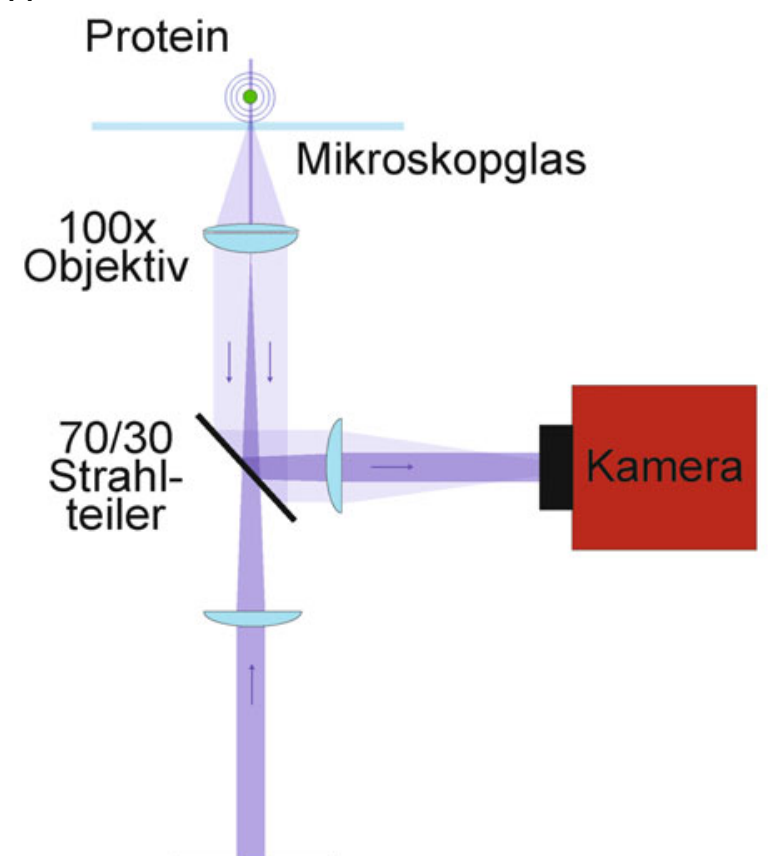

$450 \mathrm{~nm}$ Laser
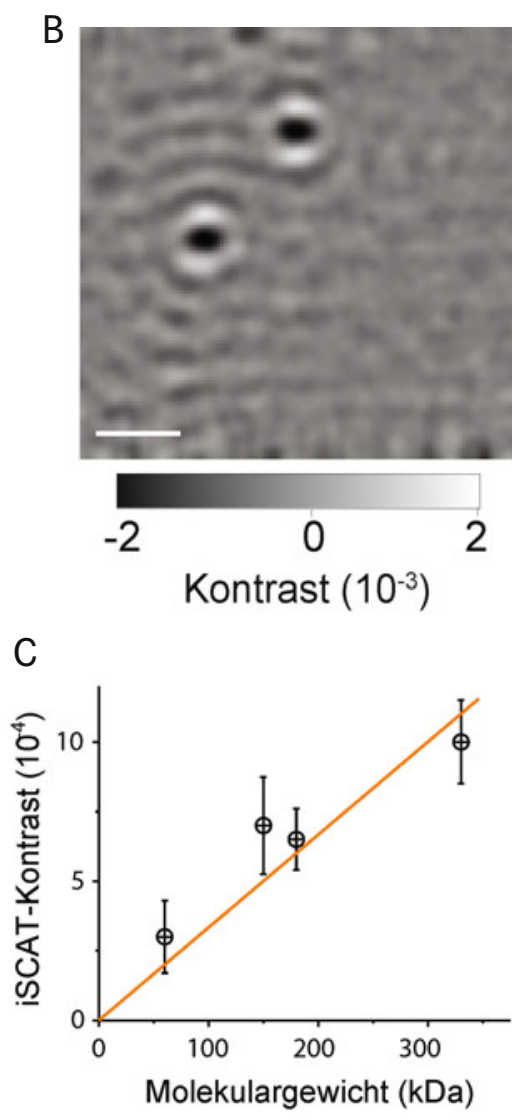

4 Abb. 1: Prinzip der isCAT-Mikroskopie. A, Aufbau: Weitfeldbelichtung der Probenoberfläche mit einem Laser, Aufsammeln des teilweise reflektierten Lichts sowie eines Teils des vom Teilchen gestreuten Lichts mit dem Objektiv und Umlenkung auf einen Kamerasensor. B, von der Kamera registriertes Interferenzsignal zweier einzelner Proteine. Maßstab: $1 \mu \mathrm{m}$ (aus [8]). C, Der iSCATKontrast skaliert linear mit der Masse des Proteins (aus [5]). 
vierten Mikroskopie unumgänglich. Jedoch führt das Einbringen eines Markers zwangsläufig zu einem Eingriff in den biologischen Prozess und zu einem gewissen Filter, der Proteine, die nicht markiert und vielleicht nicht bekannt sind, buchstäblich unerkannt im Dunkeln lässt. Die Sensitivität der genannten Methoden liegt in der Regel weit oberhalb von einzelnen Molekülen und erfordert häufig den Einsatz von ganzen Ensembles von Zellen, um eine zur Detektion ausreichende Menge an Proteinen zur Verfügung zu stellen. Dies steht jedoch oft im Widerspruch zu dem Wunsch, Messungen an einzelnen Zellen vornehmen zu können, um fundamentale Prozesse leichter zu isolieren und verstehen $\mathrm{zu}$ können. Das Ziel sind also Techniken, die in der Lage sind, ganz ohne den Bedarf an Markern einzelne Proteine in Echtzeit aufzulösen.

In den vergangenen Jahren wurden verschiedene Methoden in diese Richtung entwickelt. Die Verwendung von komplexen nano-optischen Techniken, basierend auf verschiedenen Technologien, wie oberflächen- verstärkte Raman-Spektroskopie (SERS), Plasmonik oder Mikroresonatoren, erreichen heute die nötige Empfindlichkeit für die Detektion einzelner Proteine [1]. Diese Methoden beruhen jedoch - neben der Notwendigkeit von Oberflächenfunktionalisierung - auf der elektromagnetischen Feldverstärkung in einem sehr kleinen Sensorbereich und erfordern zudem oft eine statistische Interpretation des Signals.

Dabei ist es durchaus möglich, einzelne Proteine direkt zu visualisieren, und zwar einzig über das Streusignal, das bei der Beleuchtung eines solchen Moleküls (etwa mit einem Laser) entsteht. Dieses an sich sehr schwache Signal kann mittels Interferenz detektiert werden, indem man es mit einem Referenzsignal überlagert und damit verstärkt. Die interferometrische Detektion von gestreutem Licht, kurz iSCAT (interferometric detection of scattered light), wurde 2004 in unserer Gruppe entwickelt [2] und unter anderem zur Detektion von Viren auf einer Membran genutzt [3]. Die Methode wird seither stetig weiterentwickelt und findet sich mittlerweile - in teils abgewandelter Form und verschiedensten Anwendungsbereichen - in den Experimenten zahlreicher Gruppen weltweit wieder [4].

Die Detektion gestreuten Lichts wird auch in der Dunkelfeldmikroskopie angewendet. Die Grundidee ist simpel: Das zum Beleuchten der Probe verwendete Licht wird nicht zum Auge bzw. der Kamera weitergeleitet, sondern abgeblockt, etwa durch eine Blende im Mikroskop oder eine schräg einfallende Beleuchtung. Lediglich das an der Probe gestreute Licht erreicht den Detektor. Hier zeigt sich jedoch ein fundamentales Problem: Der Streuquerschnitt und damit die Intensität des gestreuten Lichts skaliert mit dem Quadrat der Polarisierbarkeit des Teilchens. Diese ist wiederum abhängig vom Brechungsindex des Teilchens relativ zu dem seiner Umgebung sowie vom Teilchenvolumen. Da Teilchen mit einem Durchmesser, der deutlich kleiner als die Wellenlänge des Lichts ist, hier als Kugel betrachtet werden können, zeigt sich, dass ein Teilchen mit einem Durchmesser von fünf Nano- 

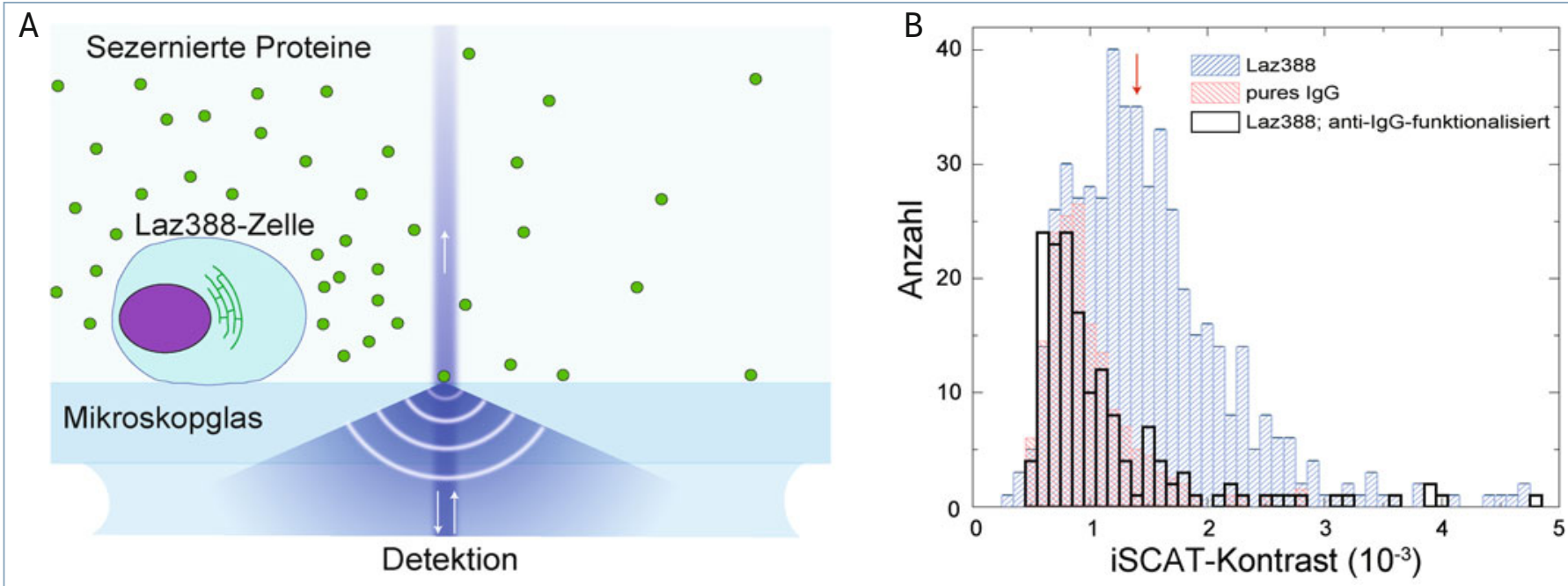

A Abb. 2: Prinzip der Zellsekretionsmessung mit iSCAT. A, Platzierung einer einzelnen, lebenden Zelle nahe der iSCAT-Sensorfläche. B, Histogramme der detektierten Proteine (Anzahl: 503) einer Zellmessung über 125 Sekunden auf einer nicht-funktionalisierten Oberfläche (blau) sowie auf einer antiIgG-funktionalisierten Oberfläche (schwarz) im Vergleich zu reinem IgG auf einer nicht-funktionalisierten Oberfläche (rot) (aus [8]).

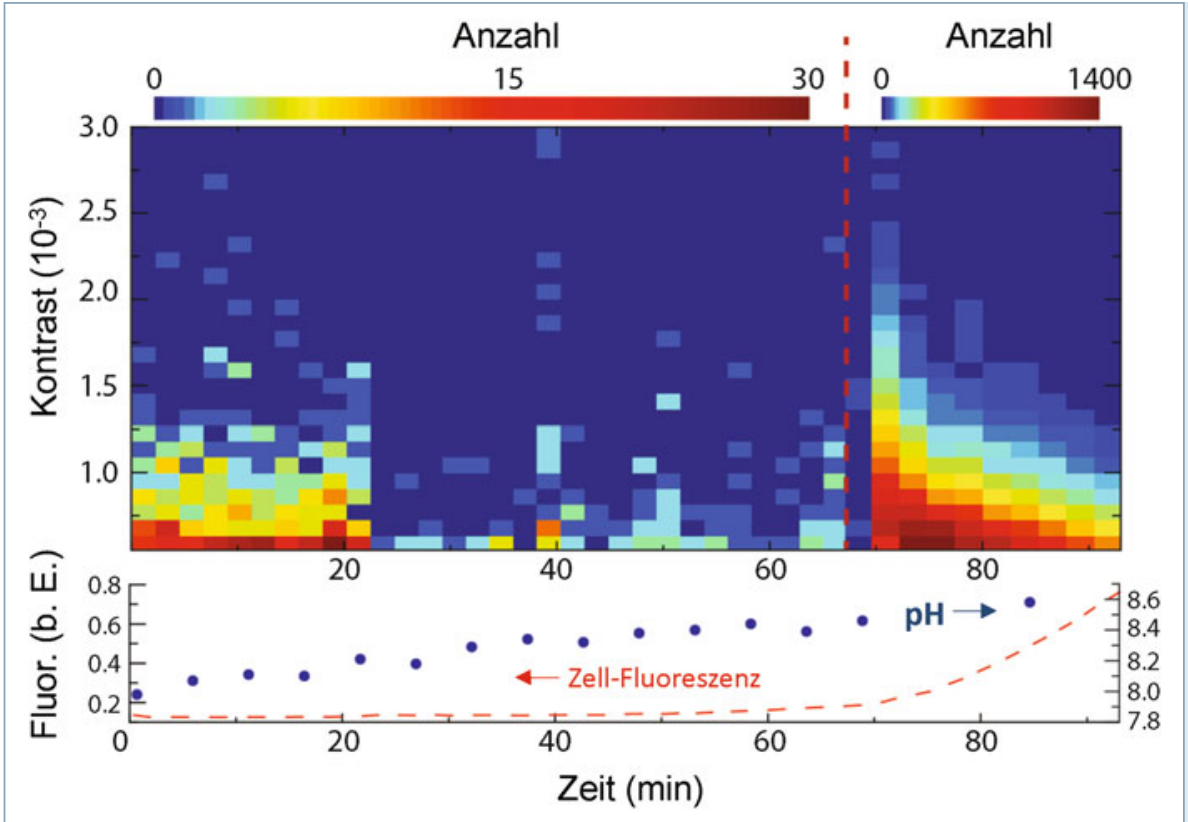

metern (etwa ein Protein) im Vergleich zu einem Teilchen mit 50 Nanometer Durchmesser (etwa ein Virus) tatsächlich eine Million Mal weniger Licht streut. Damit fällt das Streusignal eines Proteins weit unter das detektierbare Niveau in der Dunkenfeldmikroskopie.

Um das mit sinkender Teilchengröße drastisch abfallende Streusignal noch detektieren zu können, wird es in der iSCAT-Mikroskopie nun mit einer Referenzwelle zur Interferenz gebracht. Gleichung 1 zeigt die Intensität am Detektor:

$I_{\text {det }} \propto\left|\vec{E}_{r e f}+\vec{E}_{s}\right|^{2}=$

$\left|\vec{E}_{r e f}\right|^{2}+\left|\vec{E}_{s}\right|^{2}+2\left|\vec{E}_{r e f}\right|\left|\vec{E}_{s}\right| \cos \theta$.
Hier ist $\vec{E}_{r e f}$ das elektrische Feld der Referenzwelle und $\vec{E}_{\mathrm{s}} \propto \alpha$ das elektrische Feld der vom Teilchen gestreuten Welle, welches wie oben genannt von der Polarisierbarkeit $\alpha$ und dem elektrischen Feld des auf das Teilchen einfallenden Lichts $\vec{E}_{i}$ abhängt. Das für iSCAT relevante Interferenzsignal ist der dritte Term in Gleichung 1. Er enthält neben den Feldern $\vec{E}_{r e f}$ und $\vec{E}_{S}$ (man beachte die lineare Abhängigkeit vom gestreuten Feld und damit von der Polarisierbarkeit) noch einen Term $\cos \theta$, der vom Phasenunterschied der beiden beteiligten Wellen herrührt. Das Interferenzsignal skaliert also nur linear mit dem Volumen und überwiegt damit das quadratisch skalierende Streusignal für kleine Teilchen.
Abb. 3: Sekretionsdynamik einer einzelnen, lebenden Zelle unter stetigem Anstieg des pHWertes des umgebenden Mediums. Drei unterschiedliche Bereiche kennzeichnen die Sekretion einer intakten Zelle, einer gestressten Zelle unter erhöhtem $\mathrm{pH}$-Wert und die Detektion von Teilen des Zellinneren auf der Sensoroberfläche nach Eintreten des Zelltods und Auflösen der Zellmembran (verändert nach [8]).

Der Grundaufbau von iSCAT ist in Abbildung 1A dargestellt; mithilfe eines Lasers und eines Objektivs wird die Probenoberfläche beleuchtet, wobei das einfallende Licht teilweise an der Grenzfläche zwischen Mikroskopglas und Probenflüssigkeit reflektiert wird und als Referenzwelle agiert. Befindet sich nun beispielsweise ein Protein in der Nähe dieser Grenzfläche, streut es das einfallende Laserlicht in alle Richtungen. Ein Teil dieses Streusignals wird von dem Objektiv eingesammelt und über einen Strahlteiler auf einen Kamerasensor gelenkt. Hier interferiert es mit der Referenzwelle und bildet das iSCATSignal. Ein typisches iSCAT-Mikroskopbild ist in Abbildung 1B dargestellt und zeigt das 
beugungslimitierte Abbild eines Teilchens, dessen Kontrast von der oben erwähnten Polarisierbarkeit abhängt. In guter Näherung kann man die Dichte und den Brechungsindex verschiedener Proteine als gleich betrachten, sodass einzig die Teilchenmasse als bestimmender Parameter für den iSCATKontrast bleibt. Diesen linearen Zusammenhang kann man nutzen, um das iSCAT-Signal für verschiedene Proteine zu kalibrieren. Dies wurde erstmals 2014 von M. Piliarik et al. [5] demonstriert, die Proteine bis zu einer Teilchengröße von 60 Kilodalton (Albumin) nachgewiesen haben (Abb. 1C). Im Jahr 2018 verbesserten G. Young et al. [6] die Signalanalyse und zeigten die Detektion von Proteinen bis zu 50 Kilodalton, mit einer Auflösung von 19 Kilodalton und einer Massengenauigkeit von zwei Prozent, die es ihnen unter anderem erlaubte, unterschiedliche Liganden in einem Streptavidin-Biotin-System voneinander zu unterscheiden.

Ein weiterer interessanter Aspekt dieser Mikroskopiemethode ist die Tatsache, dass das iSCAT-Signal (im Gegensatz zur Fluoreszenz) nicht gesättigt werden kann; je größer die Lichtintensität des einfallenden Lichts, desto größer ist auch die gestreute und somit die detektierte Intensität. Theoretisch ermöglicht dies also die Detektion beliebig kleiner Moleküle bzw. das beliebig schnelle Tracking von Molekülen mit hinreichend großem Signal. Zusätzlich stellt das Hintergrundrauschen der verwendeten Kameras im Gegensatz zur Detektion schwacher Signale in Dunkelfeld- oder Fluoreszenzmikroskopie kein Problem dar, da das Gesamtsignal in iSCAT durch die Mischung mit dem Referenzfeld beliebig angehoben werden kann. In der Praxis wird iSCAT schließlich durch die technischen Eigenschaften der Lichtquelle und der Kamera sowie durch die mechanische Stabilität des Aufbaus limitiert (weitere, detaillierte Informationen finden sich in [4]).

Zusätzlich enthält das iSCAT-Signal neben dem zentralen Kontrast noch eine weitere, sehr interessante Information: Das Beugungsbild eines Teilchens entsteht durch die Interferenz zweier Wellenformen, zum einen die vom Teilchen gestreute Welle (eine Kugelwelle) und zum anderen die am Mikroskopglas reflektierte Referenzwelle (eine ebene Welle). Das Beugungsmuster enthält mehrere ringförmige Nebenmaxima und -minima, wie man es vom Beugungsmuster eines kleinen Lochs kennt. Bei genügend großem Signal erlauben deren Kontrast und Abstand einen Rückschluss auf den Abstand des Teilchens zur Referenzwelle bzw. der Oberfläche des Mikroskopglases. Damit sind Aussagen über die relative Höhenänderung des Teilchens möglich, sodass beispielsweise die Diffusion eines Proteins in drei Dimensionen möglich wird [7].

Wir haben iSCAT unter anderem für die Echtzeitdetektion und Visualisierung von Proteinen verwendet, die von einzelnen Zellen sezerniert werden [8]. Dafür verwendeten wir LAZ388-Zellen, eine Epstein-Barr-Virus-transformierte BZelllinie. Der iSCAT-Aufbau wurde in diesen Messungen um einen Hellfeld- und einen Fluoreszenzkanal erweitert, die es erlaubten, die Zellen während eines Experiments zu lokalisieren und ihre Viabilität über einen fluoreszenten Marker (Propidiumiodid) zu kontrollieren.

Für das Experiment wurden die Zellen in Puffer gewaschen und in eine Messküvette aus Plexiglas gegeben. Nachdem sie sich auf der Oberfläche des Deckgläschens abgesetzt hatten, wurde eine einzelne, lebende Zelle in der Nähe der iSCAT-Sensorfläche platziert. Ein Abstand von ca. fünf Mikrometern verhinderte, dass die Zelle direkt von dem Laser getroffen wurde (Abb. 2A). Anschließend wurde das iSCAT-Signal der Proteine aufgenommen, die in dem vom Laser beleuchteten Blickfeld auf dem Deckgläschen adsorbierten. Abbildung 2B (blau) zeigt das Histogramm der Signale über einen Zeitraum von 125 Sekunden. Der iSCAT-Kontrastbereich von 0,035 bis 0,36 Prozent entspricht einer Massenverteilung der Proteine von 100 bis 1.100 Kilodalton. Dieser bereits erwähnte lineare Zusammenhang kann über Referenzmessungen an bekannten Proteinspezies hergestellt werden [5]. Dabei ist zu beachten, dass diese Messung ohne das Markieren von Proteinen durchgeführt wurde und ein weites Spektrum der sezernierten Proteine bzw. deren Massen zeigt. Aus diesen kann man einzelne Spezies genauer untersuchen, indem man beispielsweise die Oberfäche des Deckgläschens funktio- 
nalisiert, etwa mit Antikörpern, die gegen Immunglobulin $\mathrm{G}$ ( $\mathrm{IgG}$ ) gerichtet sind, welches von LAZ388 sezerniert wird. Wiederholt man die iSCAT-Messung so, zeigt sich das schwarze Histogramm in Abbildung 2B, dessen Position und Verteilung mit der in einer Messung von purem IgG (rotes Histogramm) übereinstimmt.

Ein besonderer Vorteil unserer Methode ist die Möglichkeit, Informationen in Echtzeit zu erhalten. Damit kann etwa die Dynamik eines Sekretionsprozesses untersucht werden, was mit konventionellen Methoden nur sehr aufwendig möglich ist. Ein beispielhafter Verlauf (Abb. 3) der Sekretion einer "gestressten“ Zelle (unter stetigem Anstieg des pH-Wertes des umgebenden Mediums) zeigt, wie zunächst zahlreiche Proteine mit Massen von bis zu 400 Kilodalton sezerniert werden. Nach ca. 20 Minuten nimmt die Menge der detektierten Proteine deutlich ab und nach weiteren 50 Minuten wieder sprunghaft zu; in diesem Moment war die Zellmembran so weit permeabilisiert, dass Teile des Zellinneren auf dem iSCAT-Messbereich registriert wurden.

Diese Ergebnisse verdeutlichen das Potenzial der iSCAT-Mikroskopie für die Messung von Sekretionsprozessen individueller, lebender Zellen. Die Methode ermöglicht die Visualisierung einzelner, unmarkierter Proteine mit einer Zeitauflösung von Millisekunden und einem vergleichsweise einfachen optischen Aufbau (eine detaillierte Video-Anleitung zur Durchführung dieser Messungen findet sich in [9]). Weitere, sehr interessante Projekte für diese Art von iSCAT-Messungen wären beispielsweise die Untersuchung der Kommunikation mehrerer Zellen miteinander (z. B. zwischen einer T-Zelle und einer Antigen-präsentierenden Zelle) sowie die systematische Untersuchung des Einflusses äußerer Parameter wie Temperatur oder von Signalstoffen auf die Sekretionsdynamik einzelner oder mehrerer Zellen.

\section{Literatur}

[1] Xavier J, Vincent S, Meder F et al. (2018) Advances in optoplasmonic sensors - combining optical nano/microcavities and photonic crystals with plasmonic nanostructures and nanoparticles. Nanophotonics 7:1-38

[2] Lindfors K, Kalkbrenner T, Stoller P et al. (2004) Detection and spectroscopy of gold nanoparticles using supercontinuum white light confocal microscopy. Phys Rev Lett 93:037401 [3] Kukura P, Ewers H, Müller C et al. (2009) High-speed nanoscopic tracking of the position and orientation of a single virus. Nat Methods 6:923-927

[4] Taylor RW, Sandoghdar V (2019) Interferometric scattering microscopy: seeing single nanoparticles and molecules via Rayleigh scattering. Nano Lett 19:4827 4835

[5] Piliarik M, Sandoghdar V (2014) Direct optical sensing of single unlabelled proteins and super-resolution imaging of their binding sites. Nat Commun 5:4495

[6] Young G, Hundt N, Cole D et al. (2018) Quantitative mass imaging of single biological macromolecules. Science 27:423-427

[7] Taylor RW, Gholami Mahmoodabadi RG, Rauschenberger $\mathrm{V}$ et al. (2019) Interferometric scattering microscopy reveals microsecond nanoscopic protein motion on a live cell membrane. Nat Photonics 13:480-487

[8] McDonald MP, Gemeinhardt A, König K et al. (2018)

Visualizing single-cell secretion dynamics with single-protein sensitivity. Nano Lett 18:513-519
[9] Gemeinhardt A, McDonald MP, König K et al. (2018) Label-free imaging of single proteins secreted from living cells via iSCAT microscopy. J Vis Exp (141), doi: $10.3791 / 58486$

Open Acces:

This article is distributed under the terms of the Creative Commons Attributio 4.0 International License (http://creativecommons.org/licenses/by/4.0/), which permits use, duplication, adaption, distribution and reproduction in any medium or format, as long as you give appropriate credit to the original author(s) and the source, provide a link to the Creative Commons license, and

Open access funding provided by Max Planck Society

\section{Korrespondenzadresse:}

Prof. Dr. Vahid Sandoghdar

Max-Planck-Institut für die Physik des Lichts und Max-Planck-Zentrum für Physik und Medizin Staudtstraße 2

D-91058 Erlangen

Tel.: 09131-7133 301

vahid.sandoghdar@mpl.mpg.de

www.mpl.mpg.de

Katharina König
Studium der Angewandten Naturwissenschaft an der TU Bergakademie Freiberg. Seit
2014 Doktorandin bei Prof. Dr. V. Sandoghdar am Max Planck Institut für die Physik des
Lichts in Erlangen.

